\section{Reply to S.A. Upadhyaya}

We thank Upadhyaya ${ }^{1}$ for his comments on our article, Reddy et $\mathrm{al}^{2}{ }^{2}$ and his questions regarding the relevance of craniospinal irradiation (CSI) to the outcomes reported. ACNSO333 (ClinicalTrials.gov identifier: NCT00653068) was a nonrandomized trial that prescribed intensive chemotherapy and radiation therapy (RT), and the results were analyzed according to the intent-to-treat principle. The study was designed to identify whether the therapeutic regimen increased survival. ACNS0333 was powered for the primary analysis, namely the comparison of risk of an event-free survival (EFS) for patients younger than age 36 months at diagnosis with cohorts of patients from historical cooperative groups. ${ }^{2}$ The examination of other variables, including RT, was exploratory with no expectation of demonstrating conventional statistical significance. The goal of that article was to present primary and secondary objective results.

The sequence of induction chemotherapy, consolidation chemotherapy, and RT was based on the age of the patient at the completion of induction chemotherapy, the location of the primary tumor, and the extent of disease at the time of enrollment (Reddy et al, ${ }^{2}$ Table A1). RT timing, dose, and treatment volumes were defined in the protocol by the ACNSO333 study and Children's Oncology Group (COG) Radiation Therapy Committees, on the basis of an understanding of treatment toxicities for this young patient population. CSI was recommended for patients who had disseminated disease at enrollment, but ultimately, it was given at the discretion of the treating institution. Upadhyaya ${ }^{1}$ made three major points that we respond to here.

First, to address the questions raised regarding possible differences in outcome based on the type of RT, we will provide descriptive survival analysis for the seven patients younger than age 3 years with metastatic disease who received treatment, including RT, on protocol (Table 1). Three patients received focal RT only and all are alive with no EFS events. Four patients received CSI plus a focal RT boost. Two of these patients had EFS events: both had progressive disease after RT. One patient died of disease and the other patient remains alive after additional therapy. Given the very small number of patients in each of these categories, we feel that further statistical analysis of these data would not be meaningful. However, these data are being combined with other data in a metaanalysis (Aridgides, personal communication). Three patients with metastatic disease who received chemotherapy on study but no RT because of physician or parent preference have all died of disease. As stated in our article, the presence of metastasis did not significantly increase the risk of death on the basis of the data presented in Tables 3 and 4 in Reddy et al. ${ }^{2}$ In Table A1 in that article, we delineated that CSI was recommended for patients with metastatic disease, and in Figure 2, we showed that six of 40 patients who received $\mathrm{RT}$ also received CSI. We do not feel that we have misled the reader on this point; rather, we presented the data so that readers could draw their own conclusions. In the Discussion, we mentioned that additional details on delivery of RT and related changes will be reported separately.

Second, the timing of RT was based on safety, with the goal of placing it as early as possible in the treatment scheme. We noted in the article that timing of RT did not affect survival for patients who continued to receive protocol therapy past induction and recommended that, in future trials, RT follow completion of all chemotherapy because of two treatment-associated deaths that occurred in patients who received RT between induction and consolidation. As mentioned earlier, CSI was given at the discretion of the treating institution, and although this along with other variables could be considered independently, the number of patients in any

TABLE 1. Patients Younger Than Age 36 Months With Metastatic Disease, RT, and Survival

\begin{tabular}{llccccccc} 
Patient & $\begin{array}{c}\text { Primary } \\
\text { Tumor } \\
\text { Location }\end{array}$ & $\begin{array}{c}\text { Metastatic } \\
\text { Status } \\
\text { at Diagnosis }\end{array}$ & $\begin{array}{c}\text { Age at } \\
\text { Enrollment } \\
\text { (months) }\end{array}$ & Focal RT & CSI & EFS (years) & OS (years) & Alive \\
\hline 1 & Infratentorial & M3 & 10.99 & Yes & No & 6.99 & 6.99 & Yes \\
\hline 2 & Infratentorial & M3 & 16.51 & Yes & Yes & 3.74 & 3.75 & No (DOD) \\
\hline 3 & Infratentorial & M2 & 11.41 & Yes & No & 5.27 & 5.27 & Yes \\
\hline 4 & Infratentorial & M2 & 7.30 & Yes & No & 4.21 & 4.21 & Yes \\
\hline 5 & Supratentorial & M2 & 11.28 & Yes & Yes & 4.92 & 4.92 & Yes \\
\hline 6 & Supratentorial & M3 & 14.34 & Yes & Yes & 3.68 & 3.68 & Yes \\
\hline 7 & Infratentorial & M3 & 16.94 & Yes & Yes & 0.88 & 3.46 & Yes
\end{tabular}

Abbreviations: CSI, craniospinal radiation; DOD, died of disease; EFS, event-free survival; M2, gross nodular seeding of brain; M3, gross nodular seeding of spine; OS, overall survival; RT, radiation therapy. 
one category is simply too low to perform meaningful statistical analyses.

Finally, we performed an intent-to-treat analysis that included all evaluable patients. Once a patient comes off protocol therapy, COG does not require sites to provide additional treatment data and does not require that data be submitted to the Imaging and Radiation Oncology Core. Patients who came off protocol therapy may have received a variety of treatments, so analysis of these data, even if they were available, would be uninterpretable. An analysis that excludes patients who came off therapy for parent or physician choice without having an EFS event is planned. As stated in the article, data from ACNS0333 should be combined with that from other studies to more clearly identify prognostic factors and help risk-stratify patients in future trials. Aridgides et al have recently performed a metaanalysis of radiation and response to chemotherapy for patients with metastatic atypical teratoid/rhabdoid tumor. ACNS0333 patients who received radiation on protocol are included in this analysis, which will be presented at the 2020 American Society for Radiation Oncology meeting.

\section{Alyssa T. Reddy, MD}

University of California San Francisco, CA

\section{Mark D. Krailo, PhD}

Keck School of Medicine, University of Southern California, Los Angeles, CA

\section{Allen B. Buxton, MS}

Children's Oncology Group, Monrovia, CA

\section{Douglas R. Strother, MD}

Cumming School of Medicine, University of Calgary, Calgary, $A B$, Canada

Annie Huang, MD, PhD

Arthur and Sonia Labatt Brain Tumour Research Centre, Hospital for Sick Children, Toronto, ON, Canada

\section{Tianni Zhou, PhD}

California State University at Long Beach, Long Beach, CA

\section{Alexander R. Judkins, MD}

Children's Hospital Los Angeles, Keck School of Medicine, University of Southern California, Los Angeles, CA

Peter C. Burger, MD

Johns Hopkins University, Baltimore, MD
Ian F. Pollack, MD

University of Pittsburgh Medical Center, Children's Hospital of Pittsburgh, Pittsburgh, PA

Chris Williams-Hughes, BS

Children's Oncology Group, Monrovia, CA

\section{Maryam Fouladi, MD}

Cincinnati Children's Hospital Medical Center, Cincinnati, $\mathrm{OH}$

\section{Ben Ho, MSc}

Hospital for Sick Children, Toronto, ON, Canada

Claire M. Mazewski, MD

Aflac Cancer and Blood Disorders Center, Children's

Healthcare of Atlanta, Emory University School of Medicine, Atlanta, GA

Victor A. Lewis, MD

Cumming School of Medicine, University of Calgary,

Calgary, AB, Canada

Louis-Gilbert Vezina, MD

The George Washington University School Medicine and Health Sciences, Washington, DC

Timothy N. Booth, MD

University of Texas Southwestern, Dallas, TX

\section{Anita Mahajan, MD}

Mayo Clinic, Rochester, MN

\section{AUTHORS' DISCLOSURES OF POTENTIAL CONFLICTS OF INTEREST AND DATA AVAILABILITY STATEMENT}

Disclosures provided by the authors and data availability statement (if applicable) are available with this article at DOI https://doi.org/10.1200/ JC0.20.01573.

\section{REFERENCES}

1. Upadhyaya SA: Relevance of the type and timing of radiation therapy to the outcomes reported in the ACNSO333 trial for atypical teratoid/rhabdoid tumors. J Clin Oncol 38:3352-3353, 2020

2. Reddy AT, Strother DR, Judkins AR, et al: Efficacy of high-dose chemotherapy and three-dimensional conformal radiation for atypical teratoid/ rhabdoid tumor: A report from the Children's Oncology Group Trial ACNS0333. J Clin Oncol 38:1175-1185, 2020 
AUTHORS' DISCLOSURES OF POTENTIAL CONFLICTS OF INTEREST

\section{Reply to S.A. Upadhyaya}

The following represents disclosure information provided by authors of this manuscript. All relationships are considered compensated unless otherwise noted. Relationships are self-held unless noted. I = Immediate Family Member, Inst = My Institution. Relationships may not relate to the subject matter of this manuscript. For more information about ASCO's conflict of interest policy, please refer to www.asco.org/rwc or ascopubs.org/jco/authors/author-center.

Open Payments is a public database containing information reported by companies about payments made to US-licensed physicians (Open Payments).

Alyssa T. Reddy

Consulting or Advisory Role: Novartis
Mark D. Krailo

Consulting or Advisory Role: Merck Sharp \& Dohme

Travel, Accommodations, Expenses: Merck Sharp \& Dohme

No other potential conflicts of interest were reported. 